\title{
Non-destructive X-Ray testing of complex mechanisms and devices
}

\author{
Ali Ozdiev ${ }^{1, *}$, Yury Kryuchkov ${ }^{1}$, and Hans-Michael Kroning ${ }^{2}$ \\ ${ }^{1}$ Tomsk Polytechnic University, 634050 Tomsk, Russia \\ ${ }^{2}$ Saarland University, Saarbrücken, D-66123, Germany
}

\begin{abstract}
X-ray tomography becomes a wide-spread non-destructive testing method. Flexibility of the reconstruction algorithms makes possible to apply 3D tomographic analysis to complex mechanisms and systems and detect defects of different types. For instance, this opportunity allows to solve geometrical problem, when the size of the investigating sample exceeding dimensions of the setup. This paper proposes to use nonstandard geometry of tomographic scanning and backprojection algorithm optimized for this case to solve previously mentioned problem. Producing and assembling of complex space systems and mechanics requires the testing procedure at each step of the technological process: for separate parts of mechanism as well as for assembled system or device. Presented approach prospectively fits for this task.
\end{abstract}

\section{Introduction}

One of the most important advantages of CT measurements is the opportunity to obtain detailed 3D visualization of the sample including its internal structure, which is the way to detect defects: cracks, pores, splits etc. The standard geometry of the tomographic scanning (Figure 1) requires full or limited coverage of the sample by the beam in condition of matching the optical axis of the detector with the center of the sample rotation [1]. Then the standard filtered backprojection algorithm becomes applicable for the reconstruction of the certain part of the sample depending on the type of beam: parallel, fan or cone.

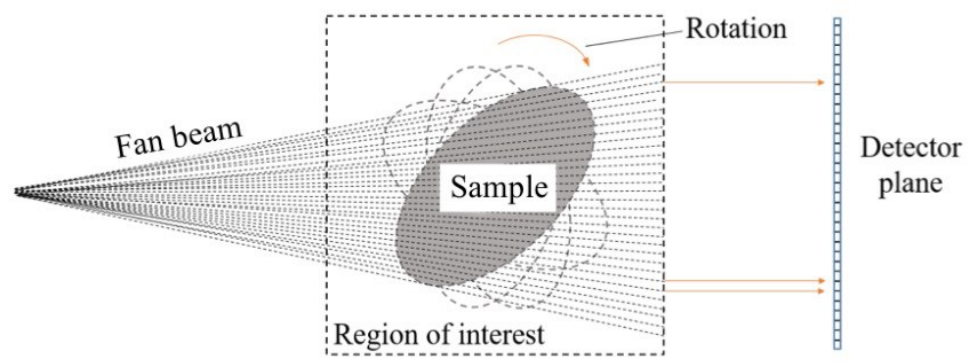

Fig. 1. The standard geometry of tomographic scanning, fan beam case.

\footnotetext{
* Corresponding author: ozdiev@tpu.ru
} 
Approaches of the tomographic scanning of the object with sizes, which are bigger, than the dimensions of X-ray setup are still developing. Optimization and adaptation of filtered backprojection algorithms are the way of building the X-ray setup for the scanning of such objects.

\subsection{Scanning the objects along their longest dimension}

Step-shift scanning was proposed to scan objects with sizes exceeding dimensions of the Xray setup. Straight forward way to conduct such scanning is to build scanning trajectory along the longest dimension of the sample as it shown in Figure 2.

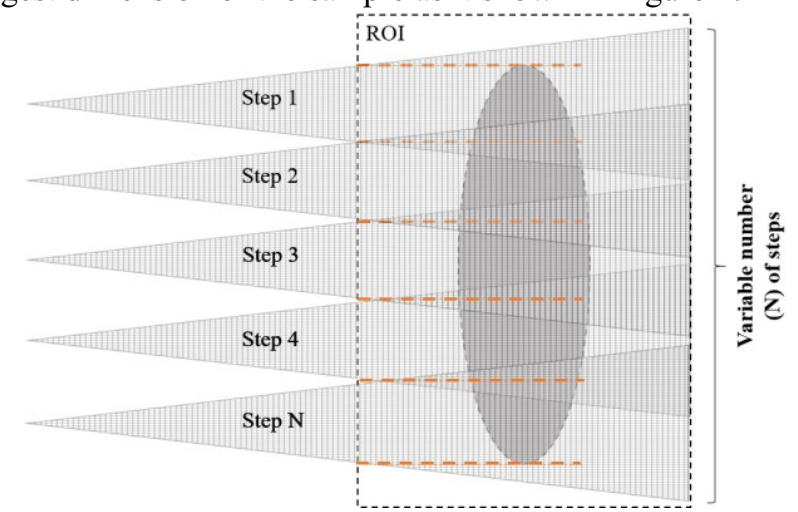

Fig. 2. Step-shift scanning of the sample along its longest view (top view).

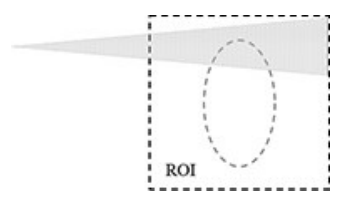

Fig. 3. Backprojection along the exact beam trajectory.

In case when the center of rotation matches with the center of the object one of conditions for applicability of standard backprojection algorithm fails for the entire scanning step except of the only step where the optical axis of the setup matches with the center of the sample. This collision requires the optimization of the backprojection algorithm by ignoring the above condition and projecting the data along the exact trajectories for each scanning step.

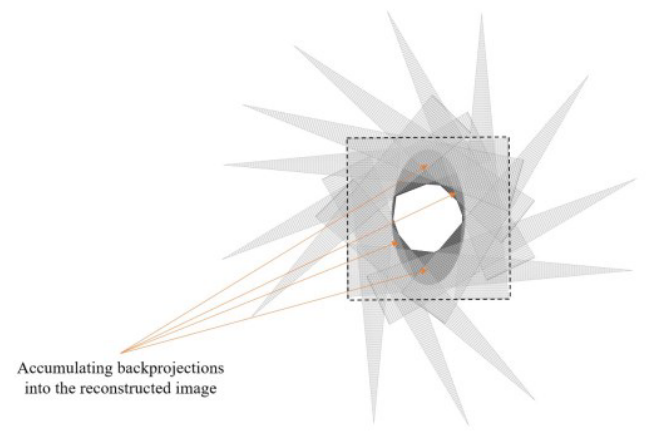

Fig. 4. Reconstruction of the object area from the single scanning step. 
Projecting the data along the exact trajectories for each scanning step means that for the certain area of the region of interest, which was covered by the beam at corresponding scanning step (Figure 3), the backprojection for every rotation angle should be obtained.

Interpolation of each value generated by the detector along the real beam trajectories in Cartesian coordinate system mathematically represents the operation of back projection [2]. Reconstructed from the scanned area of the object image is produced by summarizing of all corresponding backprojections (Figure 4).

\section{Simulation and reduction of artifacts}

Collecting all the reconstructed areas from each scanning step gives opportunity to obtain totally reconstructed image of the object simply by summarizing them to each other. Socalled raw reconstructed image (Figure 5), which is the sum of separately reconstructed areas, will contain an artifact - significant intensity difference between internal and external areas of the image.
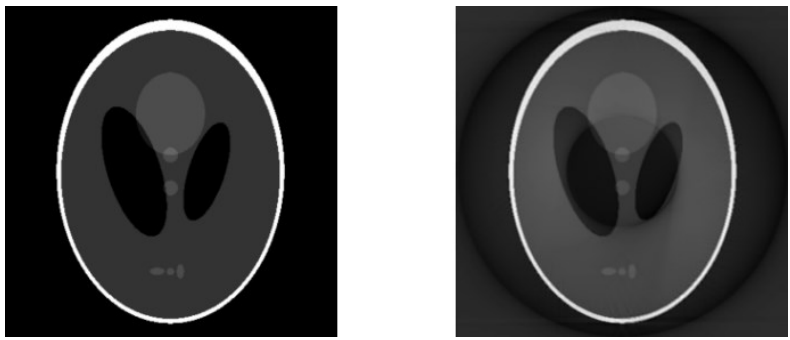

Fig. 5. Original image on the left and reconstructed image on the right with the intensity artefact.
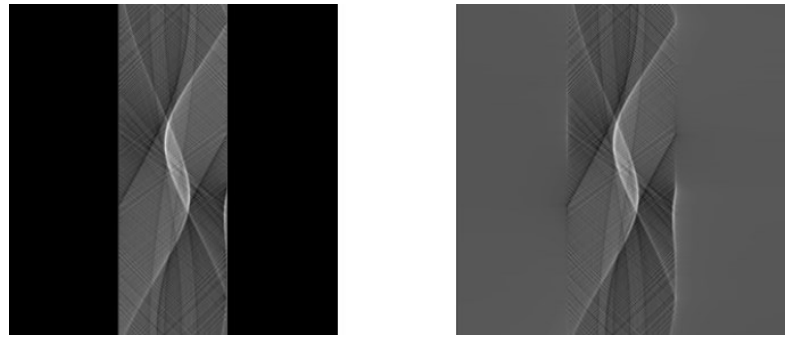

Fig. 6. The sinogram with empty areas and the sinogram where those areas filled with edge values.

Well-known Shepp-Logan phantom (Figure 5, left) was used for the simulation - a standard test image created by Larry Shepp and Benjamin F. Logan for their work in 1974. It usually serves as the model of a human head in the development and testing of image reconstruction algorithms.

The filtered backprojection algorithm was optimized for step-shift scanning, so that a field of view smaller than the object being scanned. A sinogram is a plot of the projection data - horizontal axis is the beam source angle, and vertical axis is the detector number. Because of the limited field of view, certain pixel areas of a sinogram stay empty or simply equal to zero. To reduce the artifact empty pixels of limited view sinogram should be filled out by edge values as it shown in Figure 6 [3]. 
Fulfilling this condition for every step of scanning leads to the reduction of the artifact and results into the clearly reconstructed image. The reconstructed images from 3 steps of scanning and their sum in compare with the original image are presented in Figure 7.
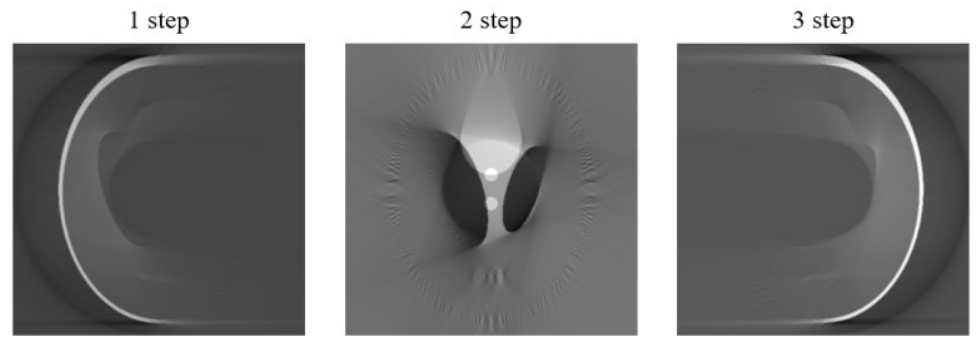

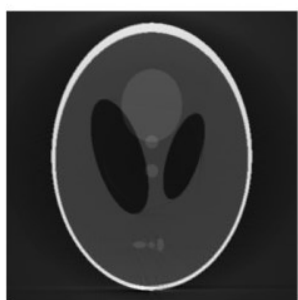

the sum

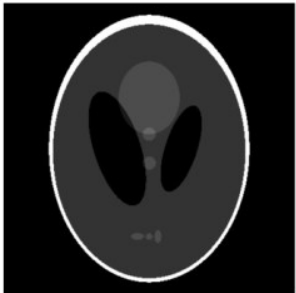

original phantom

Fig. 7. Top row shows the results of reconstruction from each of 3 steps, second row shows the sum of these steps in compare with original phantom.

\section{Conclusions}

Optimization of reconstruction algorithm is the prospective way to apply tomographic analysis for complex space systems and mechanisms as one of non-destructive control techniques. Development of new scanning geometries based on such algorithms makes possible to build real-time testing system.

The reported study was partially supported by the Governmental program "Science", research project No. 11.6342.2017/БЧ

\section{References}

1. G.T. Herman, Fundamentals of Computerized Tomography: Image Reconstruction from Projections (Springer Publishing Company, New York 2009)

2. A.C. Kak, M. Slaney, Principles of Computerized Tomographic Imaging (SIAM, Philadelphia 2001)

3. F.E. Boas, D. Fleischmann, JMI 4(2), 229 (2012) 